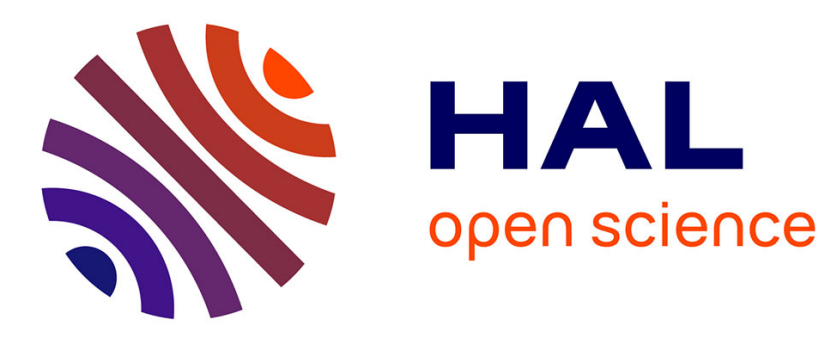

\title{
Free standing double walled boron nanotubes
}

Ali Sebetci, Ersen Mete, Ihsan Boustani

\section{To cite this version:}

Ali Sebetci, Ersen Mete, Ihsan Boustani. Free standing double walled boron nanotubes. Journal of Physics and Chemistry of Solids, 2009, 69 (8), pp.2004. 10.1016/j.jpcs.2008.02.014 . hal-00540538

\section{HAL Id: hal-00540538 \\ https://hal.science/hal-00540538}

Submitted on 27 Nov 2010

HAL is a multi-disciplinary open access archive for the deposit and dissemination of scientific research documents, whether they are published or not. The documents may come from teaching and research institutions in France or abroad, or from public or private research centers.
L'archive ouverte pluridisciplinaire HAL, est destinée au dépôt et à la diffusion de documents scientifiques de niveau recherche, publiés ou non, émanant des établissements d'enseignement et de recherche français ou étrangers, des laboratoires publics ou privés. 


\section{Author's Accepted Manuscript}

Free standing double walled boron nanotubes

Ali Sebetci, Ersen Mete, Ihsan Boustani

PII: $\quad$ S0022-3697(08)00057-7

DOI: $\quad$ doi:10.1016/j.jpcs.2008.02.014

Reference: $\quad$ PCS 5396

To appear in: $\quad$ Journal of Physics and Chemistry of Solids

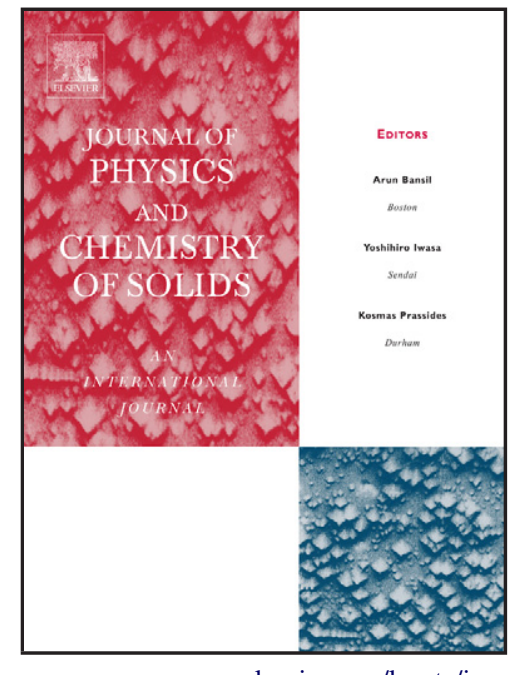

www.elsevier.com/locate/jpcs

Received date: 21 December 2007

Revised date: $\quad 4$ February 2008

Accepted date: $\quad 18$ February 2008

Cite this article as: Ali Sebetci, Ersen Mete and Ihsan Boustani, Free standing double walled boron nanotubes, Journal of Physics and Chemistry of Solids (2008), doi:10.1016/j.jpcs.2008.02.014

This is a PDF file of an unedited manuscript that has been accepted for publication. As a service to our customers we are providing this early version of the manuscript. The manuscript will undergo copyediting, typesetting, and review of the resulting galley proof before it is published in its final citable form. Please note that during the production process errors may be discovered which could affect the content, and all legal disclaimers that apply to the journal pertain. 


\title{
Free Standing Double Walled Boron Nanotubes
}

\author{
Ali Sebetci* \\ IFW Dresden, Helmholtzstraße 20, 01069 Dresden, Germany \\ Ersen Mete $^{\dagger}$ \\ Department of Physics, Balıesir University, \\ Çă̆ış Campus, Balıkesir 10145, Turkey \\ Ihsan Boustani ${ }^{\ddagger}$ \\ Bergische Universität - Gesamthochschule Wuppertal FB 9 - Theoretische Chemie, \\ Gaußstraße 20, 42097 Wuppertal, Germany
}

(Dated: February 4, 2008)

\begin{abstract}
Based on density functional calculations we propose stable structures of free standing double walled boron nanotubes in the form of two single walled boron nanotubes inside one another. Puckering of the boron sheets allows the inner atoms of the outer wall and outer atoms of the inner wall to be matched giving $s p$-type hybrid $\sigma$ bonding between the walls. The structural stability, in the case of double walled tubes, increases as the bond interaction between the walls strengthens. All the optimized structures reported in this study are electronically conducting in good agreement with the previously calculated metallic behavior of the experimentally observed single walled boron nanotubes.

PACS numbers: 73.63.Fg, 61.46.Fg, 81.07.De, 31.15.Ar, 31.15.Ew
\end{abstract}

Keywords: Boron nanotubes, DFT

\footnotetext{
*Electronic address: a.sebetci@ifw-dresden.de

${ }^{\dagger}$ Electronic address: emete@balikesir.edu.tr

${ }^{\ddagger}$ Electronic address: boustani@uni-wuppertal. de
} 


\section{INTRODUCTION}

The huge variety of crystal structures of bare boron is related to its electron deficiency from which a chemical versatility stems [1-5]. The most important among these crystal structures are the $\alpha$ - and $\beta$-rhombohedral boron where the latter is known to be thermodynamically most stable [6]. The $\alpha$-boron crystal consists of eight $\mathrm{B}_{12}$ icosahedra centered on the vertices of a rhombohedral unit cell, whereas the main structural motif in the $\mathrm{B}_{105}$ unit cell of the $\beta$-boron is a $\mathrm{B}_{84}$ polyhedron, where a central icosahedron is surrounded by twelve $\mathrm{B}_{6}$ pentagonal pyramids, and these large $\mathrm{B}_{84}$ soccer ball clusters are placed at the points of the rhombohedral lattice [7]. Although the natural phases of bulk boron do not possess laminar structures, recent experimental studies [8] confirmed the existence of quasiplanar clusters of 10-15 B atoms which have been previously predicted by first principle calculations [9]. Furthermore, single walled boron nanotubes (SWBNTs) have also been declared beforehand by Boustani and coworkers [10-13] which have also been synthesized experimentally $[14,15]$ a few years ago with a radius of nearly $18 \AA$, after the fabrication of crystalline [16] and amorphous [17] boron nanowires with diameters as small as $20 \mathrm{~nm}$. A recent review article about boron nanotubes which may serve as a basic orientation for any reader interested in pure boron chemistry can be found in [18].

It is a well-known fact that depending on their radii and chiralities, carbon nanotubes (CNTs) can be either metallic or semiconducting [19]. However, for the standard synthesis of CNTs one may achieve some control over their radii [20] but little control over their chiralities, which implies generally a rather poor control over the electronic properties of CNTs. On the contrary, it has recently been shown that all of the SWBNTs are metallic, irrespective of their radius and chiral angle [21]. Thus, a demonstration that other BNTs such as DWBNTs are also conducting independent of their radii and chiralities, one would expect to escape from the separation processes to get a better control over the electronic properties.

The present study investigates possible stable double walled boron nanotubes (DWBNTs) formed by a puckered boron sheet (PBS) which is discussed in Sec. III A by employing density functional theory (DFT). We report the structures, energies and electronic properties of DWBNTs. In the following sections after a brief summary of the methods, we will first discuss the main structural and energetic properties of the B sheets and SWBNTs and then 
the results for the DWBNTs.

\section{COMPUTATIONAL METHOD}

DFT calculations have been performed by using version 4.6 of the VASP program [22] which uses plane wave basis sets and a supercell approach to model solid materials, surfaces, and clusters [23]. We have employed the projector augmented wave (PAW) method [24, 25] to describe the electron-ion interactions. The exchange and correlation energies were considered using the generalized gradient approximation (GGA) with Perdew, Burke, and Ernzerhof (PBE96) [26] functional. We have used Monkhorst-Pack scheme for $k$-point sampling in the reciprocal space [27]. The Brillouin zone integrations were carried out over 7 and $56 k$ points in the full zone for geometry optimizations and for electronic structures, respectively. We have chosen an augmented wave cutoff energy of $415 \mathrm{eV}$ to expand the single electron Kohn-Sham wave functions. The dimensions of the supercell in the perpendicular directions

to the tubes axis were chosen as about $25 \AA$ to eliminate interactions between neighboring images. Calculations were deemed converged when changes in the interatomic forces were less than $0.01 \mathrm{eV} / \AA$. The band curves were obtained by broadening discrete energy levels on the grid of $k$ points using Gaussian smearing functions with a width of $0.1 \mathrm{eV}$. A conjugategradient algorithm [28] was used to simultaneously relax the internal coordinates and the lattice parameters. These choices ensure a good convergence of the total energy for the boron structures. The calculated binding energies per atom $\left(E_{b}\right)$ and interatomic distances for the $\mathrm{B}_{2}$ dimer, $\alpha$-boron crystal, $\mathrm{B}$ sheets, and single walled $\mathrm{B}$ nanotubes (SWBNTs) were compared with the experimental values and previous calculations in Table I. Different perspective views of the PBS, and the SWBNTs are given in Figs. 2 and 3, respectively. $E_{b}$ as a suitable measure for the structure stability is defined as the following:

$$
E_{b}=\left(n E_{1}-E_{n}\right) / n=E_{1}-E_{n} / n
$$

where $E_{1}$ is the atomic energy, $E_{n}$ is the total energy per unit cell and $n$ is the number of atoms in the unit cell.

Following Kuntsmann and Quandt [21], we have used the rectangular primitive cell, whose lattice vectors are shown in Fig. 2, to characterize boron nanotubes instead of previously used triangular [29] and honeycomb-derived [30] primitive cells, since it may describe the 
puckering of the B sheet more properly. One should note that the rectangular primitive cell contains two B atoms. All figures were produced by the XCrySDen graphics program [32].

\section{RESULTS AND DISCUSSION}

\section{A. Properties of B Sheets and SWBNTs}

As in the case of carbon nanotubes, ideal BNTs can be constructed by rolling up a stable single boron sheet akin to a single graphene sheet. In the previous theoretical calculations four different laminar structures have been discussed basically for the elemental boron: a flat regular hexagonal (graphene like) sheet [29, 31], a flat triangular sheet [21, 29-31] which is referred as the $\{1212\}$ sheet in Ref. [31] (see Fig. 1a in the present work), a flat non-regular hexagonal $\{1221\}$ sheet [31] (Fig. 1b in the present work), and an out of plane puckered sheet [21, 29-31] (Fig. 1c in the present work). We have calculated the parameters of all these sheets as well as the parameters of some other reference boron structures and listed them in Table I which are all in good agreement with both previous theoretical [21, 29-31] and experimental [33-35] studies. As described by Evans et al. [29], the flat regular hexagonal boron sheet leads to an unstable low binding energy structure, since the three valence electrons of boron $\left(2 s^{2} 2 p^{1}\right)$ cannot fill all the bonding molecular orbital states constructed by the four $2 s 2 p$ atomic states, which is not similar to the case of carbon graphene. Although the binding energy $(5.97 \mathrm{eV})$ of the flat triangular plane (Fig. 1a) is not as low as that of the hexagonal sheet, not only the degeneracy in the three possible $\sigma$-bond directions of the structure [29] but also the fact that it does not obey the Aufbau principle [9], which states that stable boron clusters can be constructed from only two basic pentagonal pyramidal $\mathrm{B}_{6}$ and hexagonal pyramidal $B_{7}$ units, suggest that the plane would be unstable with respect to puckering which breaks the triangular symmetry. In the hexagonal $\{1221\}$ structure (Fig. 1b), the three fold degeneracy of the $\sigma$-bonds is removed and two different main types of bonding are established: the localized directional two-center $\sigma$-bonds, and the delocalized three center mixed $\sigma-\pi$ bonds. Thus, the binding energy of this structure $(6.03 \mathrm{eV})$ is higher than that of the triangular one. On the other hand, when the puckered boron sheet (PBS) is considered, it can be seen in Fig. 1c that the most of the electron density forms strong $\sigma$-like bonds between the atoms lying on the same line and puckering makes the structure 
more agreeable to the Aufbau principle. Consequently, it is more stable $(6.18 \mathrm{eV})$ than the others.

As in the most of the other DFT calculations, the calculated binding energy $(6.68 \mathrm{eV})$ of the $\alpha$-boron crystal is $0.87 \mathrm{eV}$ higher than the experimental value since GGA methods generally overestimate the energy, while the experimental values of the lattice parameters are reproduced accurately. Therefore, the present results can be improved quantitatively as the available methods are improved. In our calculations, while the interatomic distance $d_{1}(1.61 \AA)$ is very close to the other theoretical calculations, the $d_{2}(1.88 \AA)$, and thus, the puckering height $h(0.92 \AA)$ are a bit longer than the previous results (see Fig. 2). We differentiate the atoms which are located on the two different parallel planes of the PBS where the planes are separated by the puckering height, by calling the first group as up and the second group as down atoms.

Most recently, the structure and energetics of the zigzag and armchair SWBNTs have been reported in Ref. [21] and those of chiral SWBNTs in Ref. [30]. The comparison of the results calculated in this work for the $(6,0)$ zigzag (it is referred as $(6,6)$ in Ref. [29]), for the $(6,6)$ chiral (it is referred as $(4,4)$ in Ref. [30]) and for the $(0,12)$ armchair tubes with corresponding previous calculations can be found in Table I. The pictures of the $(6,0)$, $(6,6)$, and $(0,12)$ SWBNTs are presented in Fig. 3. For the SWBNTs, the $E_{b}$ of the zigzag structure $(6.03 \mathrm{eV})$ is bigger than that of the armchair configuration $(5.92 \mathrm{eV})$ which is in good agreement with what was presented in Ref. [21]. The energy of the chiral one (5.98 $\mathrm{eV}$ ) is in between these two extremes. This can be understood in terms of the $\sigma$-bonds in the cylindrical puckered surfaces. In the zigzag surface, the direction of the $\sigma$-bonds is longitudinal and therefore, the distances $(1.62 \AA)$ between the $\mathrm{B}$ atoms on the same lines do not differ much from the one $(1.61 \AA)$ in the PBS for both of the radially inner and outer atoms which were referred as up and down atoms of the PBS. Thus, the most of the PBS's $\sigma$-bonds are conserved. However, the $\sigma$-bonds lie circumferentially in the armchair surface, and since the distance between the outer atoms on the same circumferential lines are stretched, some of the binding energy is lost. Therefore, the $E_{b}$ of the armchair structure is less than that of the zigzag one. Another trend can be seen in the difference of the inner $\left(r_{1}\right)$ and outer $\left(r_{2}\right)$ radii $\left(\Delta r=r_{2}-r_{1}\right)$, which corresponds to the puckering height $(h)$ of the PBS. They are $0.21 \AA, 0.85 \AA$, and $1.41 \AA$, for zigzag, chiral, and armchair B nanotubes, respectively. The angle $\theta$ between neighboring up-down-up atoms (see Fig. 2) increases in 
zigzag surfaces, and decreases in armchair surfaces. The puckering height $(h=0.92 \AA)$ of the PBS is more resembled in the chiral nanotube $(\Delta r=0.85 \AA)$. Any deviation of the angle $\theta$ from its equilibrium value in the PBS results in a loss of energy due to the multicentered bonds between up and down atoms which are weaker than the $\sigma$-bonds. A chiral $(k, l)$ structure, where the integers $k$ and $l$ are relatively prime and the ratio $l T_{2}^{2} / k T_{1}^{2}$ is an integer, can conserve the most of the interatomic distances and angles of the PBS (a more detailed description of this statement can be found in Appendix A of the Ref. [21]). In our calculations, $\left|T_{1}\right|=2.87 \AA$ and $\left|T_{2}\right|=1.61 \AA$ for the precision of the first two decimal points. Thus, the least relatively prime $k$ and $l$ numbers making the above ratio an integer are 529 and 1681, respectively, in our case. Since the number of atoms in the supercell of such a nanotube will be of the order of hundred thousands, we are unable to calculate its $E_{b}$, at the moment. In addition, the radius of this chiral nanotube will be about $494 \AA$, and since the puckering height will be negligible with respect to this radius, the zigzag and armchair nanotubes with such radii will have very close $E_{b}$ to that of the chiral one which goes to the limiting energy of the PBS. Furthermore, the cylindrical shape may not be the lowest energy structure of this huge nanotube as in the case of carbon nanotubes, and they may collapse [36]. The $h_{l}$ values of the armchair and zigzag nanotubes (see Fig. 3) correspond to the lengthes of the first $\left(\left|T_{1}\right|\right)$ and second $\left(\left|T_{2}\right|\right)$ primitive lattice vectors of the PBS, respectively. Thus, the $h_{l}$ distances of $2.86 \AA$ for $(12,0)$ and $1.62 \AA$ for $(6,0)$ SWBNTs are not surprising.

\section{B. Structures and energetics of DWBNTs}

We have identified three different zigzag $(6,0)+(10,0),(6,0)+(12,0),(6,0)+(14,0)$ and three different armchair $(0,12)+(0,20),(0,12)+(0,24),(0,12)+(0,28)$ DWBNTs and presented their optimized geometric structures in Fig. 4 and Fig. 5, respectively. The $E_{b}$ and geometric parameters have been reported in Table II. The unrelaxed initial configuration of each of these nanotubes consists of two zigzag or two armchair SWBNTs. During the optimization processes, the outer atoms of the inner walls were matched to the inner atoms of the outer walls in the same layer by the interatomic forces. Thus, there are chemical bonds between the walls of these nanotubes. When the $(6,0)$ zigzag SWBNT is worn by the $(10,0)$ or $(12,0)$ nanotubes, it shrinks radially and as a result stretches longitudinally because of 
the electrostatic potential induced by the outer nanotube. The average radius of $3.21 \AA$ for the $(6,0)$ SWBNT becomes $3.03 \AA$ and $3.11 \AA$ while its $1.62 \AA h_{l}$ separation is increased to 1.69 and $1.70 \AA$ for the first and second zigzag DWBNTs, respectively. When the outer wall is replaced by the $(14,0)$ nanotube, the trend changes in such a way that the bonds between the walls along the radial direction stretches whereas the longitudinal interatomic distances do not change considerably. On the contrary, when the $(0,12)$ armchair SWBNT is covered by the $(0,20),(0,24)$ or $(0,28)$ nanotubes, the radial distances are significantly extended in each case, while the $h_{l}$ distance $(2.86 \AA)$ is increased slightly. The average radius of the $(0,12) \operatorname{SWBNT}(2.64 \AA)$ becomes $2.90 \AA, 3.43 \AA$, and $3.56 \AA$ for the armchair DWBNTs, respectively.

It has been previously shown [12] that the binding energy, $E_{b}$, rises as the diameter of a SWBNT increases. This is true for the most of the DWBNTs as well (see Table II). As the outer wall of the zigzag nanotubes are changed from $(10,0)$ to $(14,0)$, the energy increases from $6.06 \mathrm{eV}$ to $6.25 \mathrm{eV}$. Similarly, the $E_{b}$ of the first armchair DWBNT rises from a value of $6.02 \mathrm{eV}$ to $6.27 \mathrm{eV}$, when the outer wall $(0,20)$ is replaced by the one $(0,24)$. However, the energy of the $(0,12)+(0,28)$ DWBNT $(6.24 \mathrm{eV})$ is not bigger than that of the $(0,12)+(0,24)$ DWBNT, which can be related to the more symmetric structure of the latter than the former. The $E_{b}(6.06 \mathrm{eV})$ of the $(6,0)+(10,0)$ DWBNT is slightly bigger than the energy $(6.03 \mathrm{eV})$ of the $(6,0)$ SWBNT which indicates that the bonds between the walls of this nanotube are not very tight. This is also confirmed by the total valence electron density pictures shown in Fig. 4. The most of the electron density is located between the atoms on the same longitudinal lines to form the $\sigma$-bonds similar to the ones in the PBS. The $E_{b}(6.15$ $\mathrm{eV}$ ) of the $(6,0)+(12,0)$ DWBNT is a bit much bigger, since in addition to the longitudinal $\sigma$-bonds, there are relatively stronger bonds between the walls of the nanotube due to the electron density between the walls. When the $(6,0)+(14,0) \mathrm{B}$ nanotube is considered, the calculated charge distribution shows a considerable contribution between the two walls in addition to the existing electron density along the tube axis which can easily be seen in Fig. 4. Therefore, its $E_{b}$ of $6.25 \mathrm{eV}$ is the greatest one among the zigzag DWBNTs investigated in the present study.

The circumferential bonds of the $(0,12)+(0,20)$ armchair DWBNT shown in Fig. 5 are responsible for both of the bonds between the neighboring atoms on the same circumferential lines and between the neighboring atoms of the inner and outer walls. Thus, these bonds are 
multicentered and its $E_{b}(6.02 \mathrm{eV})$ is higher than the $E_{b}(5.92 \mathrm{eV})$ of the $(0,12) \mathrm{SWBNT}$. The trend that the $E_{b}$ of the zigzag SWBNTs are greater than that of the armchair tubes is still valid for the pairs of $(6,0)+(10,0)-(0,12)+(0,20)$ and $(6,0)+(14,0)-(0,12)+(0,28)$ DWBNTs. However, this trend is broken when the second zigzag and armchair DWBNTs are taken into account. The $E_{b}$ of $6.27 \mathrm{eV}$ for the $(0,12)+(0,24)$ armchair nanotube is not higher only than the binding energy $(6.15 \mathrm{eV})$ of the $(6,0)+(12,0)$ zigzag DWBNT but also than that of the $(0,12)+(0,28)$ armchair $(6.24 \mathrm{eV})$ and $(6,0)+(14,0)$ zigzag $(6.25 \mathrm{eV})$ DWBNTs. One can easily see in the electron density isosurfaces at $0.8 \mathrm{e} / \AA^{3}$ (Fig. 5) of this DWBNT that there are strong $\sigma$-like bonds in both of the circumferential and radial directions. Similar radial and circumferential bonds can also be identified in the $(0,12)+(0,28)$ DWBNT. However, the less symmetric structure of the $(0,12)+(0,28)$ tube results in a slightly less stable configuration. Because of the higher symmetry of the $(0,12)+(0,24)$ structure, the average bond length $\left(\Delta r_{w}\right)$ between the walls of this tube is the smallest $(1.71 \AA)$ that contributes to the binding energy more than the others. Consequenty, the $(0,12)+(0,24)$ armchair configuration is the most stable DWBNT investigated in the present study. We would like to discuss possible chiral DWBNTs in a future work.

\section{Electronic properties of DWBNTs}

The experimental observations have shown that the $\alpha$ and $\beta$-rhombohedral boron crystals are semiconductors [37,38]. Although the recent theoretical calculations [6] of the $\beta$-rhombohedral boron contradicts to the experimental results since the ideal unit cell of the $\beta$-rhombohedral boron $\left(\mathrm{B}_{105}\right)$ does not represent the real structure which is $\left(\mathrm{B}_{106.66}\right)$, the semiconductor character of the $\alpha$-rhombohedral boron has also been calculated theoretically [39]. Opposite to the semiconducting $\alpha$ and $\beta$-boron crystals, SWBNTs show a strong conducting character $[31,39]$.

Figure 6 depicts the total and angular momentum projected density of states (DOS) for the corresponding DWBNTs. The calculated DOS of the zigzag and armchair DWBNTs show no gap with a finite DOS at the Fermi energy $\left(E_{F}\right)$ level which confirms their metallic properties. The Kohn-Sham band structures of the DWBNTs calculated along the tube axis are shown in Fig. 7. In each of these structures there are many valence and/or conduction bands crossing the Fermi energy. Thus, DWBNTs are predicted to be metallic as in the case 
of the SWBNTs.

The energy band diagram in Figure 7 shows a strong dispersion of the bands for $(6,0)+(12,0)$ DWBNT in the vicinity of the Fermi energy starting from the $\Gamma$-point to the half way between $\Gamma$ and $X$-points which forms an opening with no available states to fill. The pocket-like structure is also observed for $(6,0)+(10,0)$ and $(6,0)+(14,0)$ zigzag DWBNTs. However, it is rather less pronounced in the case of the latter one. Higher symmetry zigzag DWBNTs generate larger pockets which both span broader range of energies and extend to the higher k-vectors. The similarities in the band diagrams for $(6,0)+(10,0)$ and $(6,0)+(12,0)$ DWBNTs come out as a characteristic of the well defined longitudinal charge distribution as shown in Figure 4. The existence of strong $\sigma$-bonds along the tube axis is common for the zigzag DWBNTs which originate from puckered boron sheets. As in the case of PBS, the buckling of the zigzag DWBNT walls induce a significant dispersion in the band structures as shown in Figure 7. This is in good agreement with the anisotropic band dispersion obtained by Lau et al. for buckled $\{1212\}$ sheet [31]. Yet, similar characteristics for the energy bands can be less explicitly seen for $(6,0)+(14,0)$ due to the contributions from the circumferential and radial charge distributions which induce many available states around the Fermi level. On the other hand, the position of the Fermi level with respect to these pockets shifts down to the lower energies as the tube diameter increases. While the Fermi energy is $0.5 \mathrm{eV}$ above the pocket in the case of $(6,0)+(10,0)$, it gets inside, close to the bottom of the pocket, for the largest outer diameter zigzag DWBNT. The smoothness of corresponding DOS structures can be considered as a result of the dispersion of the bands which stems from puckering of the tube walls, symmetry of the atomic arrangements, and the axial and circumferential bonding characteristics.

DWBNTs exhibit, dominantly, $p$-channel conductivity along the tube axis which is consistent with the $\ell$-decomposed DOS diagrams in the vicinity of the Fermi levels presented in Fig. 6. DFT results show no splitting between the occupied and unoccupied energy bands at the Fermi level. The strong overlapping interaction between $\pi$ and $\pi^{*}$-states with many crossings at the Fermi energies are shown in Fig. 7. This result suggests that $\sigma$-bonding accounts for the stability of the tubular structure rather than the conductivity. Lau et al. [31] drew a similar conclusion for the stability of PBS in agreement with our statement. It becomes more clear when one considers the binding energies per boron atom in DWBNTs which are reported in Table II. The higher BEs occur for the species which have stronger cir- 
cumferential, longitudinal and/or radial $\sigma$-bonds (see Figures 4 and 5). On the other hand, the availability of the bonding and nonbonding $\pi$ states which extend along the tubular axis is the main reason for the conducting behavior of the DWBNTs.

For $(6,0)+(10,0)$ zigzag and $(0,12)+(0,20)$ armchair DWBNTs, the $\ell$-decomposed DOS calculations show that $s$-states contribute to the conduction bands more than to the valence bands within $3 \mathrm{eV}$ neighborhood of the $E_{F}$. The rich existence of unoccupied $s$-states in the conduction bands makes these structures energetically less stable compared to other DWBNTs. Indeed, $(0,12)+(0,20)$ armchair DWBNT has the highest contribution of $s$-states to the conduction bands with respect to the $p$-states, which might explain why it is the least favorable DWBNT having the smallest BE among the DWBNTs presented in this study.

The increase in the diameter of the outer walls of the armchair DWBNTs leads to a lowering of the Fermi level with respect to the top of the conduction bands which are identified as red in Figure 7. As the outer diameter of these nanotubes increases, Fermi level shifts down into the valence and below valence bands at the $\Gamma$-point. As a result, some of the initially fully occupied valence bands become partially occupied. This shift of the Fermi level with respect to the valence and conduction peaks of the DOS can also be traced in Figure 6. That becomes clear when one considers the positioning of $E_{F}$ with respect to the two successive peaks at around $1 \mathrm{eV}$ and with respect to the first peak to the valence bands which is at about $-0.3 \mathrm{eV}$ in the case of $(0,12)+(0,28)$ armchair DWBNT. These characteristic peaks can be identified in the DOS of $(0,12)+(0,24)$ DWBNT such that the two successive conduction peaks are just above the $E_{F}$ and the valence peak is positioned close to $-1 \mathrm{eV}$. Consequently, when the diameter decreases from $(0,12)+(0,28)$ to $(0,12)+(0,24)$ DWBNT, Fermi level appears to be shifted up as if it moved in the plateau between the valence and conduction peaks.

\section{SUMMARY AND CONCLUSIONS}

In conclusion, we have studied the structural, energetic, and electronic properties of the DWBNTs by using density functional calculations employing projector augmented wave method within the GGA for the exchange and correlation. We have identified several zigzag and armchair type DWBNTs predicting that it can be possible to synthesize them experimentally. As in the case of the SWBNTs, in general, zigzag DWBNTs have slightly higher 
BEs than the armchair DWBNTs with the same number of atoms in the unit cell, and as the radii of the nanotubes increase, the BEs increase, accordingly. However, our results suggest that the most stable structure appears to be the armchair $(0,12)+(0,24)$ DWBNT with the $C_{6}$ rotational symmetry that comes out as an exception for both of these rules. The tight $\sigma$-bonds of the PBS are conserved in great extend for all of these DWBNTs. Beside the longitudinal and circumferential $\sigma$-like bonds of the zigzag and armchair DWBNTs, strong chemical interactions in the radial direction can be observed between the walls of these nanotubes for some configurations. The DOS and band structure analysis suggest that DWBNTs are metallic and therefore they can be proposed as good conductive nanotubular materials. Since the conducting behaviour of DWBNTs does not depend on their radii and chiral angle similar to SWBNTs, BNTs would be potentially good nanoconductors which do not suffer from the separation difficulties existing in CNTs.

[1] E.L. Meutterties (ed.), Boron Hydride Chemistry, Academic, New York (1975).

[2] F.A. Cotton, G. Wilkinson, C.A. Murillo, M. Bochmann, Advanced Inorganic Chemistry 6th edn, Wiley, New York (1999).

[3] K. Smith, Nature 348, 115 (1990).

[4] E.D. Jemmis, M.M. Balakrishnarajan, P.D. Pancharatna, Chem. Rev. 102, 93 (2002).

[5] Proceedings of the 13th international symposium on boron, borides, and related compounds (ISBB'99), J. Solid State Chem. 154 (special issue), 1-320 (2000).

[6] D.L.V.K. Prasad, M.M. Balakrishnarajan, E.D. Jemmis, Phys. Rev. B 72, 195102 (2005).

[7] E.D. Jemmis, D.L.V.K. Prasad, J. Solid. State Chem. 179, 2768 (2006).

[8] H-J. Zhai, B. Kiran, J. Li, L-S. Wang, Nat. Mater. 2, 827 (2003).

[9] I. Boustani, Surf. Sci. 370, 355 (1997); Phys. Rev. B 55, 16426 (1997).

[10] I. Boustani, A. Quandt, Europhys. Lett. 39, 527 (1997); Compt. Mater. Sci. 11, 132 (1998).

[11] I. Boustani, A. Quandt, E. Hernandez, A. Rubio, J. Chem. Phys. 110, 3176 (1999).

[12] I. Boustani, A. Quandt, A. Rubio, J. Solid. State. Chem. 154, 269 (2000).

[13] A. Quandt, A.Y. Liu, I. Boustani, Phys. Rev. B 64, 125422 (2001).

[14] D. Ciuparu, R.F. Klie, Y. Zhu, L. Pfefferle, J. Phys. Chem. B 108, 3967 (2004).

[15] B. Kiran, S. Bulusu, H.J. Zhai, S. Yoo, X.C. Zeng, L.S. Wang, Proc. Natl. Acad. Sci. U.S.A. 
102, 961 (2005).

[16] C.J. Otten, O.R. Lourie, M-F. Yu, J.M. Cowley, M.J. Dyer, R.S. Ruoff, W.E. Buhro, J. Am. Chem. Soc. 124, 4564 (2002).

[17] L. Cao, J. Liu, C. Gao, Y. Li, X. Li, Y.Q. Wang, Z. Zhang, Q. Cui, G. Zou, L. Sun, W. Wang, J. Phys.: Condens. Matter. 14, 11017 (2002); Y.Q. Wang, X.F. Duan, Chem. Phys. Lett. 367, 495 (2003).

[18] A. Quandt, I. Boustani, Chem. Phys. Chem. 6, 2001 (2005).

[19] R. Saito, G. Dresselhaus, M.S. Dresselhaus, Physical Properties of Carbon Nanotubes (London: Imperial College Press, 1998).

[20] Z.K. Tang, H.D. Sun, J. Wang, J. Chen, G. Li G, Appl. Phys. Lett. 73, 2287 (1998).

[21] J. Kunstmann, A. Quandt, Phys. Rev. B 74, 035413 (2006).

[22] G. Kresse, J. Furthmüller, Comput. Mater. Sci. 6, 15 (1996); Phys. Rev. B 54, 11169 (1996).

[23] M.C. Payne, M.P. Teter, D.C. Allen, T.A. Arias, J.D. Joannopoulos, Rev. Mod. Phys. 64, 1045 (1992).

[24] P.E. Blöchl, Phys. Rev. B 50, 17953 (1994).

[25] G. Kresse, J. Joubert, Phys. Rev. B 59, 1758 (1999)

[26] J.P. Perdew, K. Burke, M. Ernzerhof, Phys. Rev. Lett. 77, 3865 (1996); 78, 1396 (1997).

[27] H.J. Monkhorst, J.D. Pack, Phys. Rev. B 13, 5188 (1976).

[28] W.H. Press, B.P. Flannery, S.A. Teukolsky, W.T. Vetterling, Numerical Recipes (Cambridge University Press, New York, 1986).

[29] M.H. Evans, J.D. Joannopoulos, S.T. Pantelides, Phys. Rev. B 72, 045434 (2005).

[30] I. Cabria, M.J. Lopez, J.A. Alonso, Nanotechnology 17, 778 (2006).

[31] K.C. Lau, R. Pandey, J. Phys. Chem. C. ASAP Article, DOI: 10.1021/jp066719w (2007).

[32] A. Kokalj, Comp. Mater. Sci. 28, 155 (2003).

[33] A.D. Becke, J. Chem. Phys. 84, 4524 (1988).

[34] J. Donohue, The Structures of the Elements (Wiley, New York, 1974).

[35] C. Kittel, Introduction to Solid State Physics (Wiley, New York, 1996).

[36] G. Gao, T. Cagin, W.A. Goddard III, Nanotechnology 9, 184 (1998).

[37] F.H. Horn, J. Appl. Phys. 30, 1611 (1959).

[38] R. Schmechel, H. Werheit, J. Solid State Chem. 154, 61 (2000).

[39] I. Boustani, A. Quandt, J. A. Alonso and A. Rubio: Towards Nanostructured Materials: An 
Example of Boron Nanotubes, in : Progress in Theoretical Chemistry and Physics, Eds., J. P. Julian, J. Maruani, D. Mayou and G. Deldado-Baririo, Springer, Vol. 15, 547-556 (2006). 
TABLE I: Binding energies $E_{b}$ (eV per atom) and geometric parameters $(\AA)$ of reference B structures: Flat trigonal $\{1212\}$ sheet, flat hexagonal $\{1212\}$ sheet, puckered sheet $(\mathrm{PBS}), \mathrm{B}_{2}$ dimer, $\alpha$-boron crystal, and single walled B nanotubes (SWBNTs). $d, d_{1}$, and $d_{2}$ are the B-B bond lengths, $h$ is the puckering height of PBS (see Figs. 1 and 2), $a$ and $c$ are the lattice parameters of $\alpha$-boron, $(k, l)$ are the chirality indices, $r_{1}$ and $r_{2}$ are the radii and $h_{l}$ is described in Fig. 3.

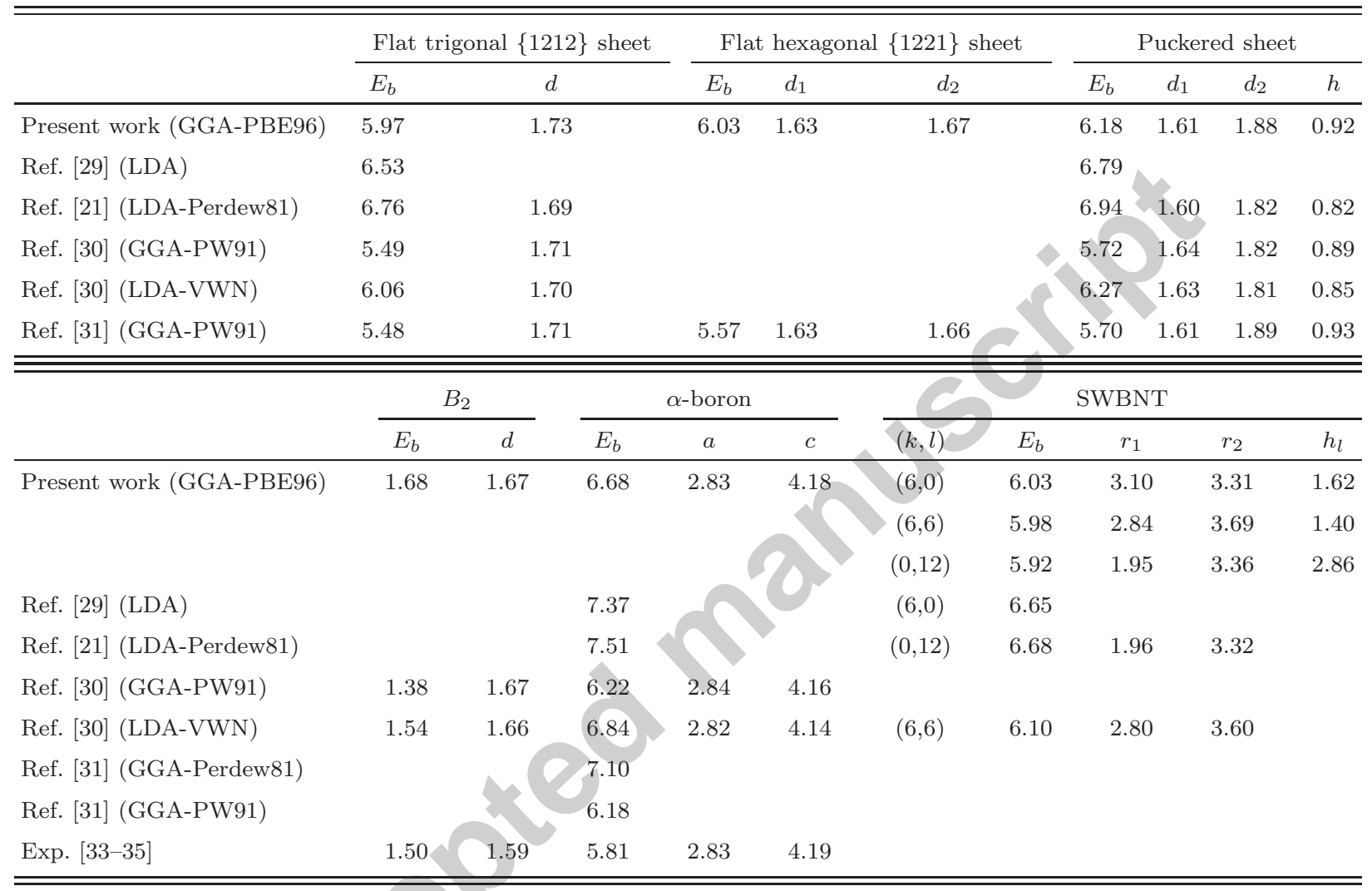

TABLE II: Binding energies $E_{b}\left(\mathrm{eV}\right.$ per atom) and geometric parameters $(\AA)$ of DWBNTs. $\left(k^{i}, l^{i}\right)$ and $\left(k^{o}, l^{\circ}\right)$ are the $k$ and $l$ indices of the inner and outer walls respectively, $n$ is the total number of atoms in the unit cell, $C_{j}$ is the rotational symmetry, $r_{1}^{i}-r_{2}^{i}$ and $r_{1}^{o}-r_{2}^{o}$ are the average innerouter radii of the inner and outer walls, $h_{l}$ is the same in Table I, $\Delta r_{w}$ is the average bond distance between the walls.

\begin{tabular}{|c|c|c|c|c|c|c|c|}
\hline$\left(k^{i}, l^{i}\right)+\left(k^{o}, l^{o}\right)$ & $n$ & $C_{j}$ & $E_{b}$ & $r_{1}^{i}-r_{2}^{i}$ & $r_{1}^{o}-r_{2}^{o}$ & $h_{l}$ & $\Delta r_{w}$ \\
\hline$(6,0)+(10,0)$ & 64 & $C_{4}$ & 6.06 & $3.03-3.03$ & $4.95-5.77$ & 1.69 & 1.84 \\
\hline$(6,0)+(12,0)$ & 72 & $C_{6}$ & 6.15 & $3.10-3.12$ & $4.97-5.86$ & 1.70 & 1.86 \\
\hline$(6,0)+(14,0)$ & 80 & $C_{2}$ & 6.25 & $3.49-3.82$ & $5.40-7.28$ & 1.63 & 1.79 \\
\hline$(0,12)+(0,20)$ & 64 & $C_{4}$ & 6.02 & $2.17-3.62$ & $5.07-6.13$ & 2.88 & 1.80 \\
\hline$(0,12)+(0,24)$ & 72 & $C_{6}$ & 6.27 & $3.05-3.81$ & $5.52-6.65$ & 2.87 & 1.71 \\
\hline$(0,12)+(0,28)$ & 80 & $C_{2}$ & 6.24 & $3.06-4.05$ & $5.82-7.62$ & 2.88 & 1.76 \\
\hline
\end{tabular}


a

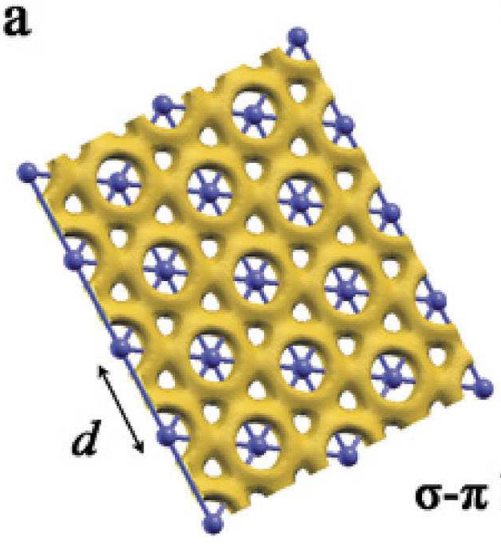

b

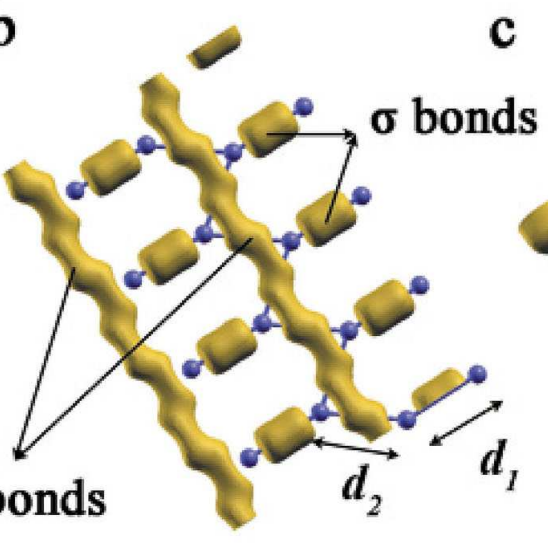

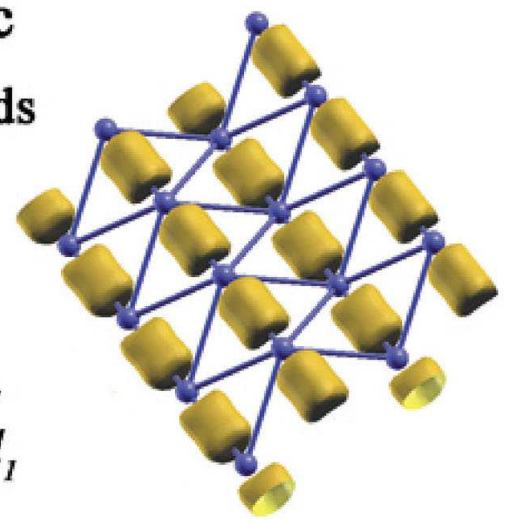

FIG. 1: (a) Flat triangular $\{1212\}$, (b) flat hexagonal $\{1221\}$ and (c) puckered boron sheets and their total valence electron density isosurfaces at $0.80 \mathrm{e} / \AA^{3}$. The ionic cores are shown as little spheres (blue online), electron densities are yellow online.
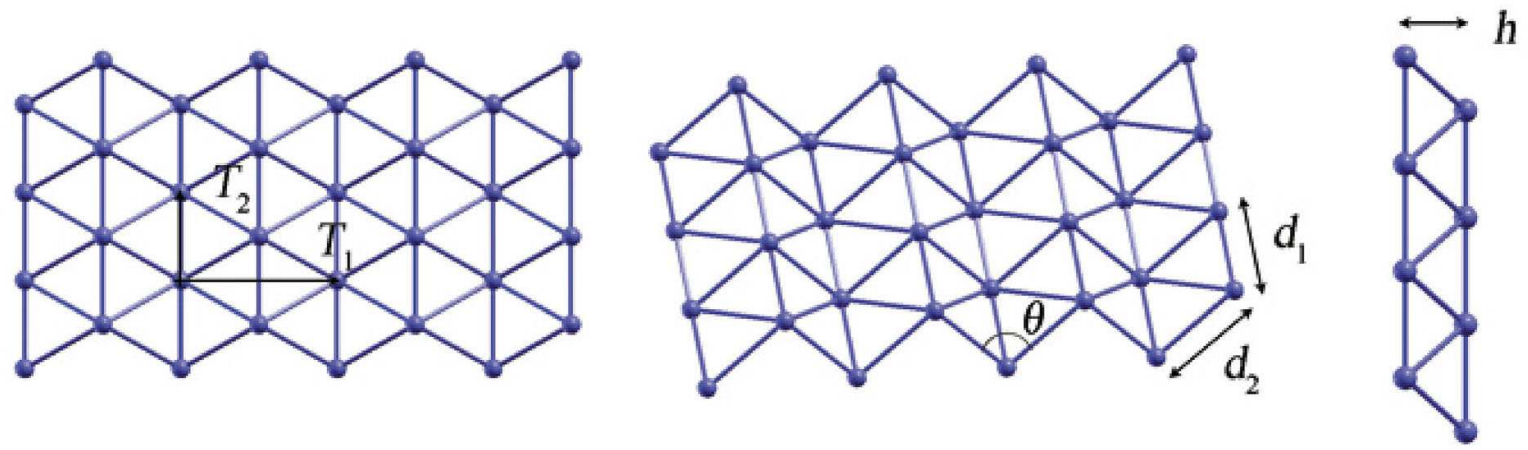

FIG. 2: Different views of the optimized puckered B sheet. $T_{1}$ and $T_{2}$ are the primitive vectors of the rectangular lattice, which are zigzag and armchair directions, respectively. 

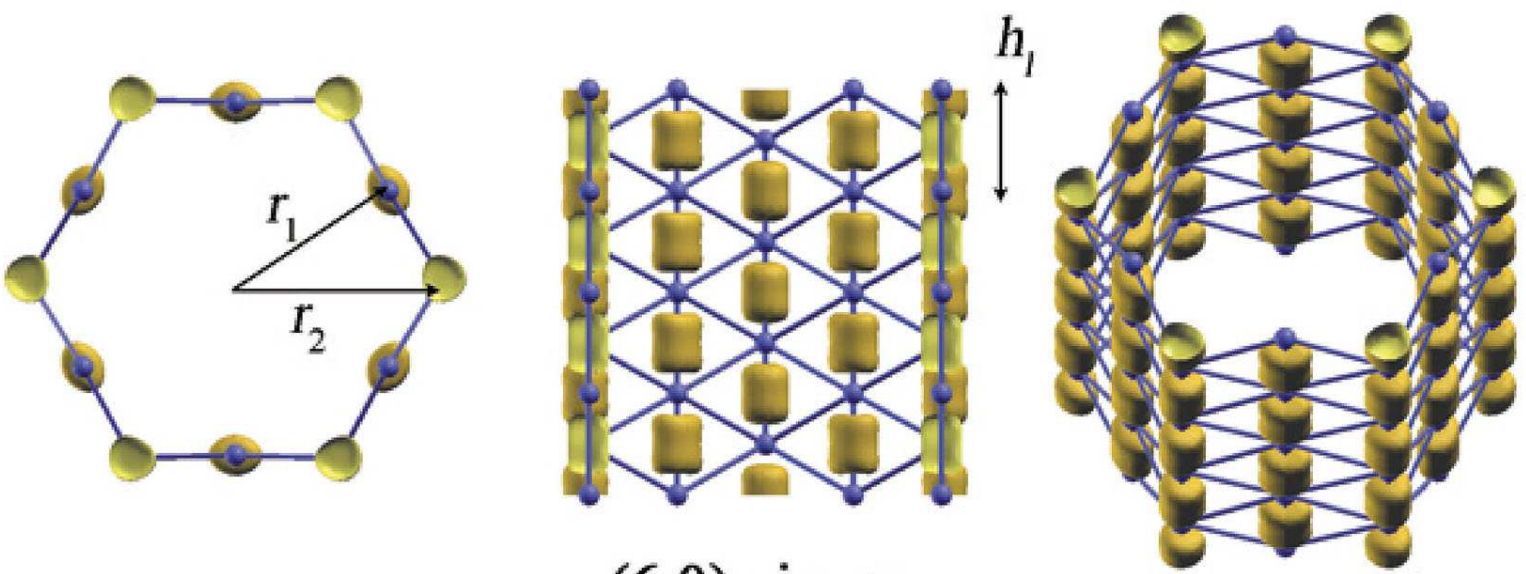

$(6,0)$ zigzag
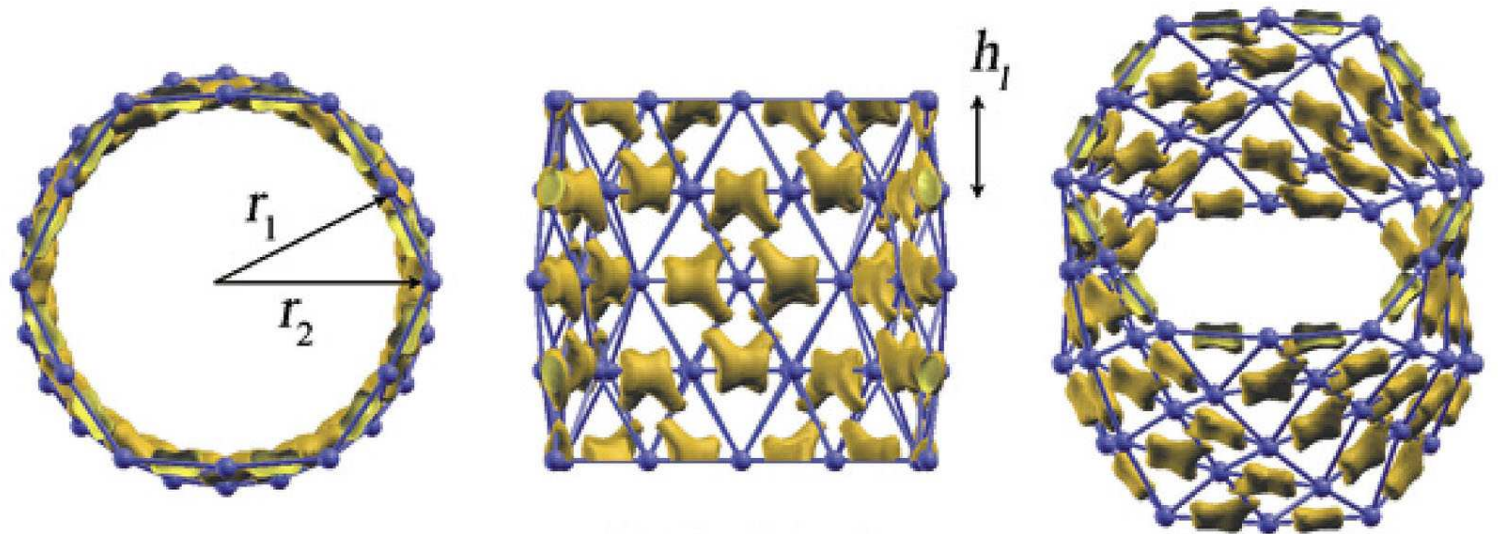

$(6,6)$ chiral
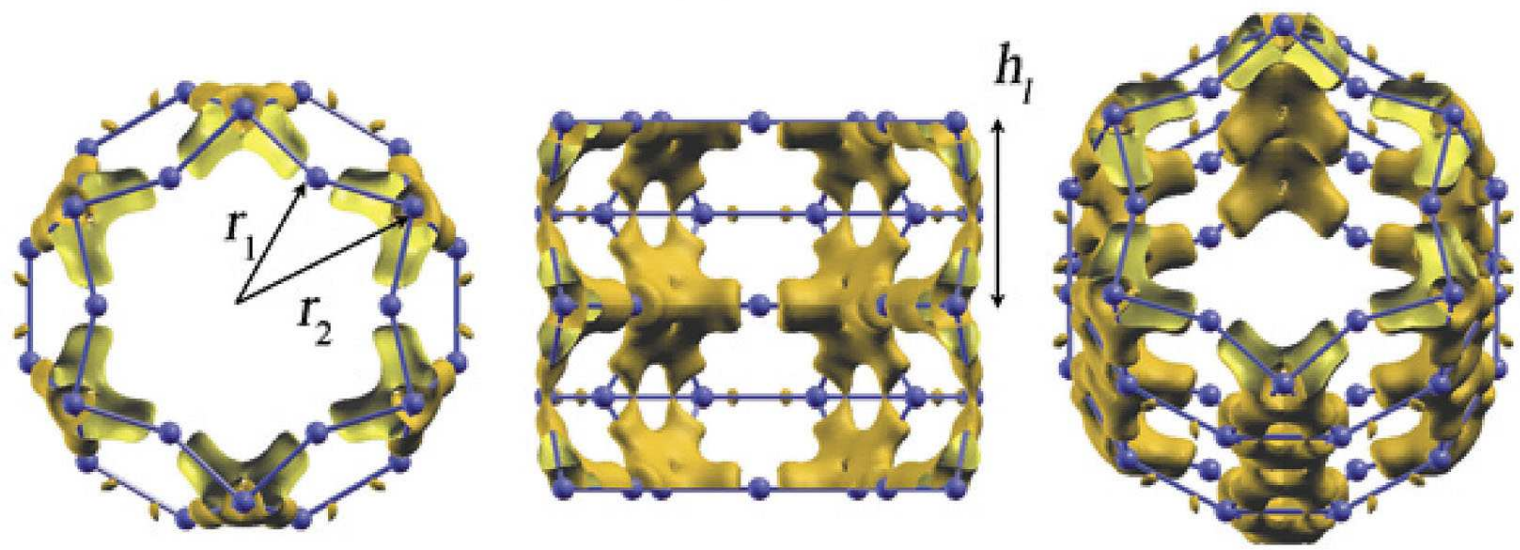

$(0,12)$ armchair

FIG. 3: Different views of the $(6,0)$ zigzag, $(6,6)$ chiral, and $(0,12)$ armchair SWBNTs and their total valence electron density isosurfaces at $0.80 \mathrm{e} / \AA^{3}$. 

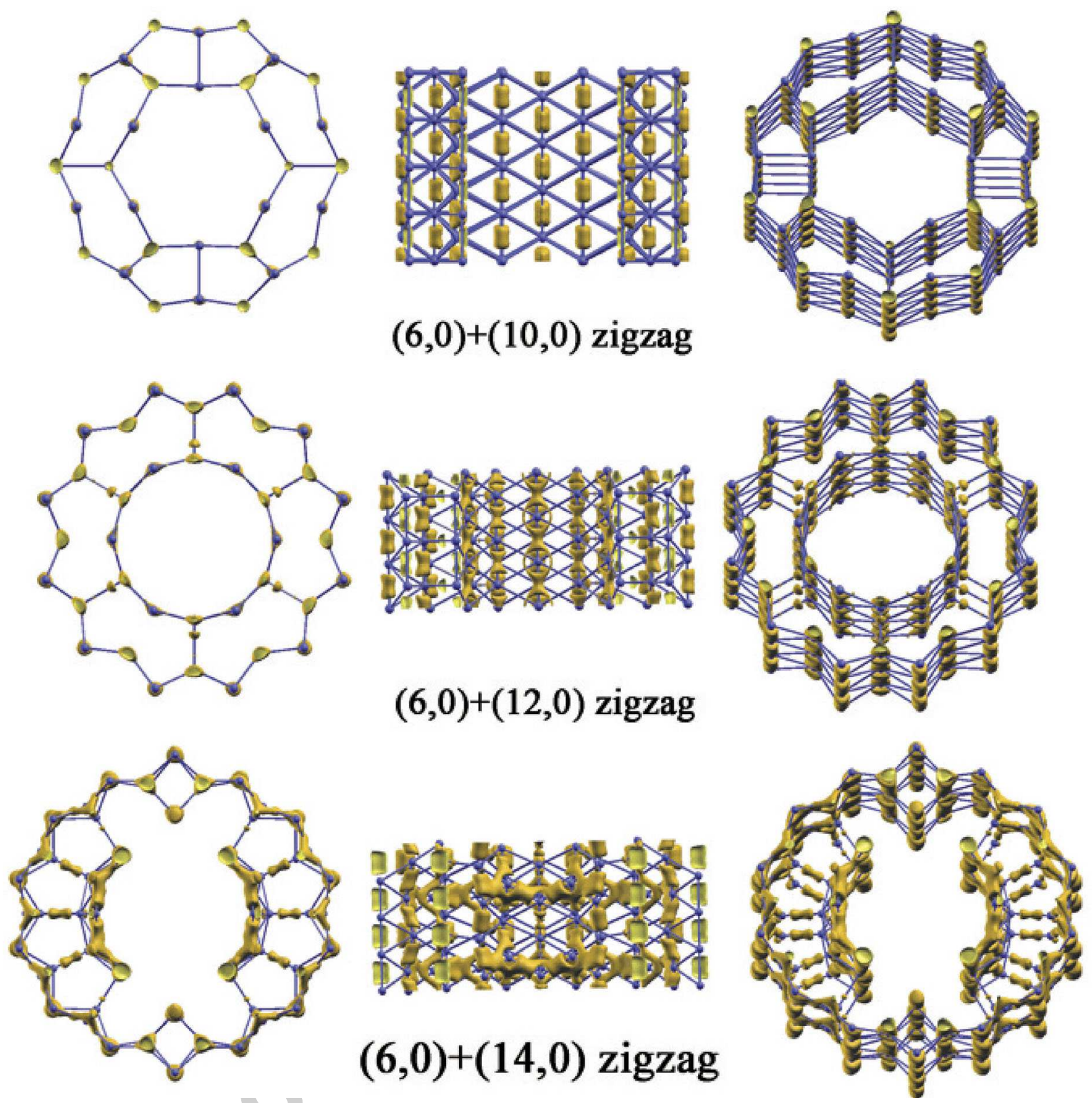

FIG. 4: Different views of the $(6,0)+(10,0),(6,0)+(12,0)$, and $(6,0)+(14,0)$ zigzag DWBNTs and their total valence electron density isosurfaces at $0.75,0.80$, and $0.80 \mathrm{e} / \AA^{3}$, respectively. 

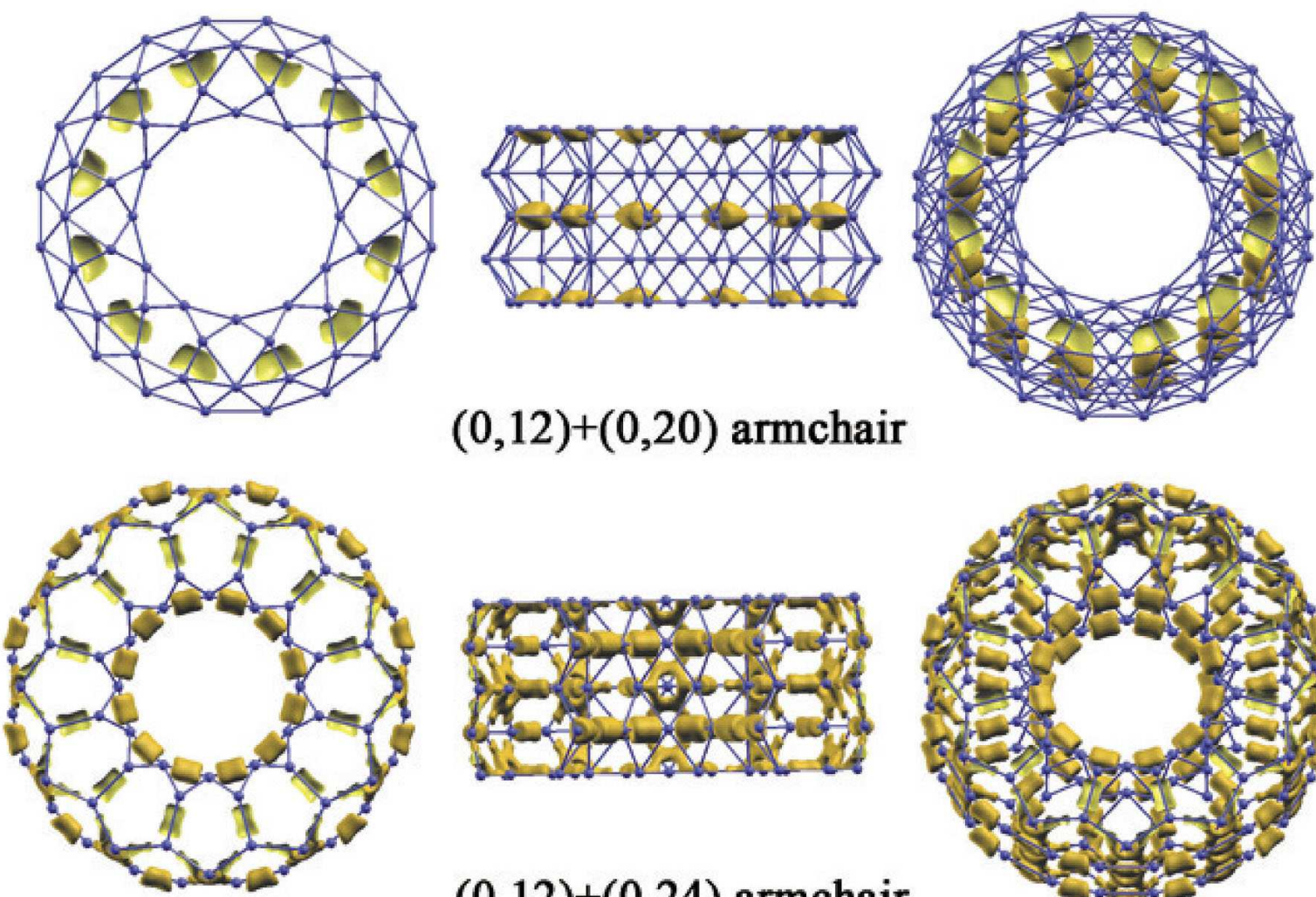

$(0,12)+(0,20)$ armchair
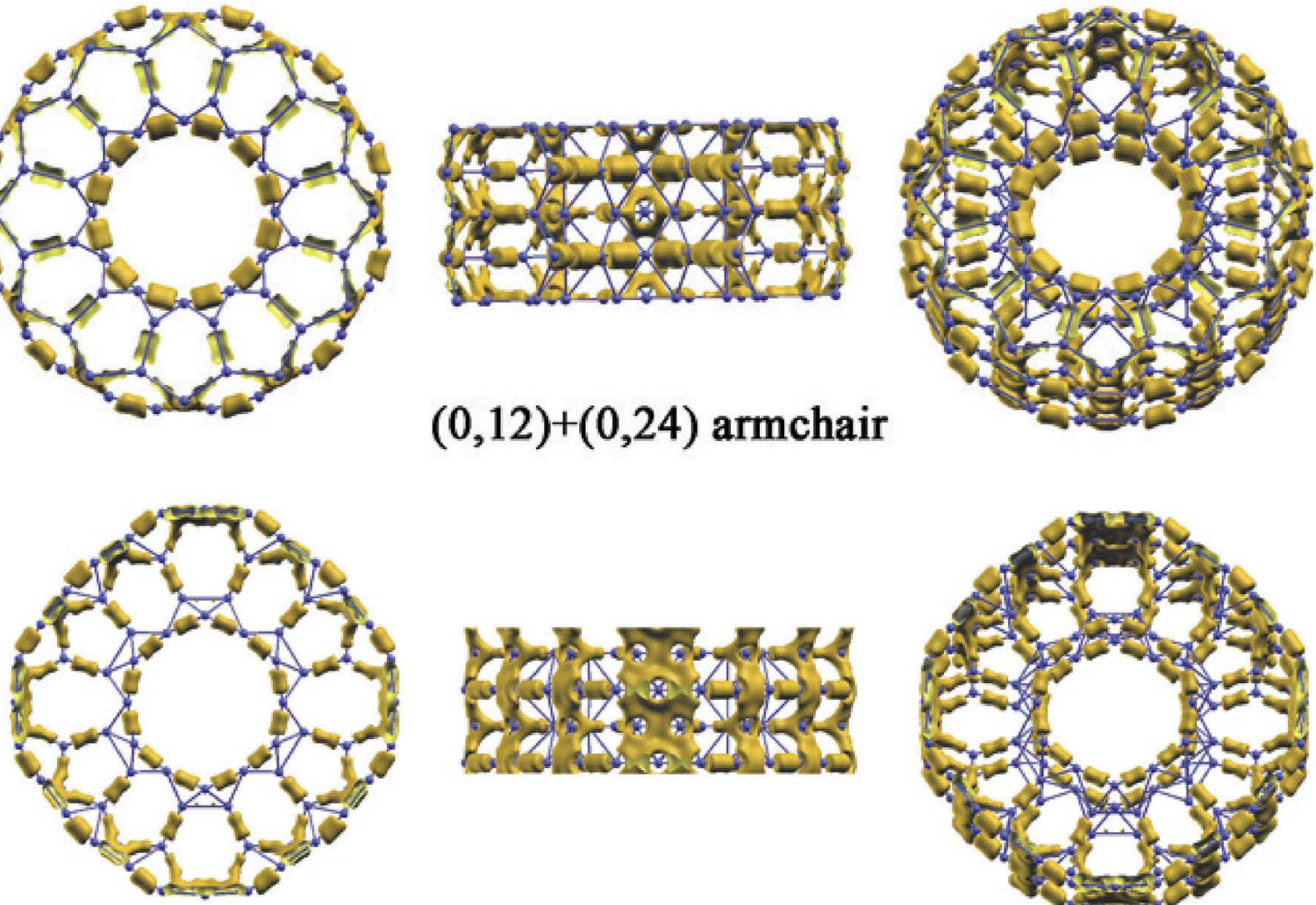

$(0,12)+(0,24)$ armchair
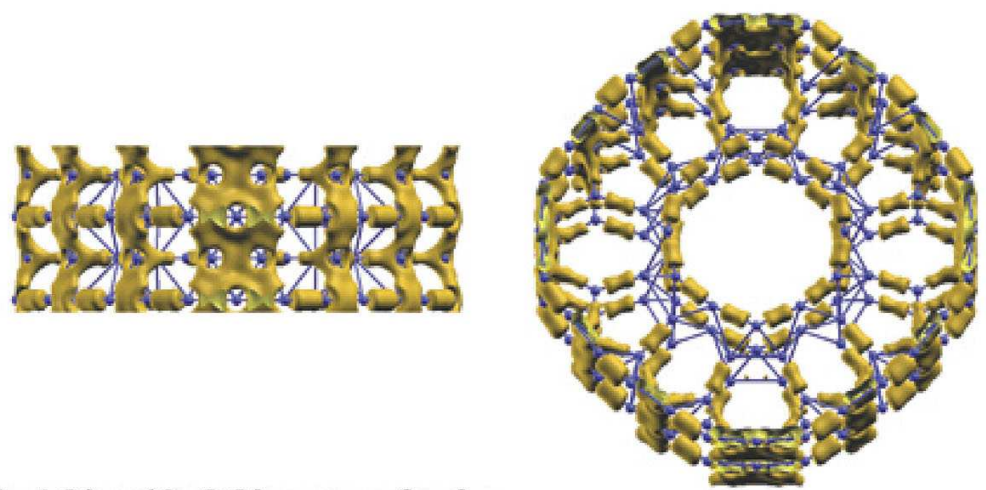

$(0,12)+(0,28)$ armchair

FIG. 5: Different views of the $(0,12)+(0,20),(0,12)+(0,24)$, and $(0,12)+(0,28)$ armchair DWBNTs and their total valence electron density isosurfaces at $0.80 \mathrm{e} / \AA^{3}$. 

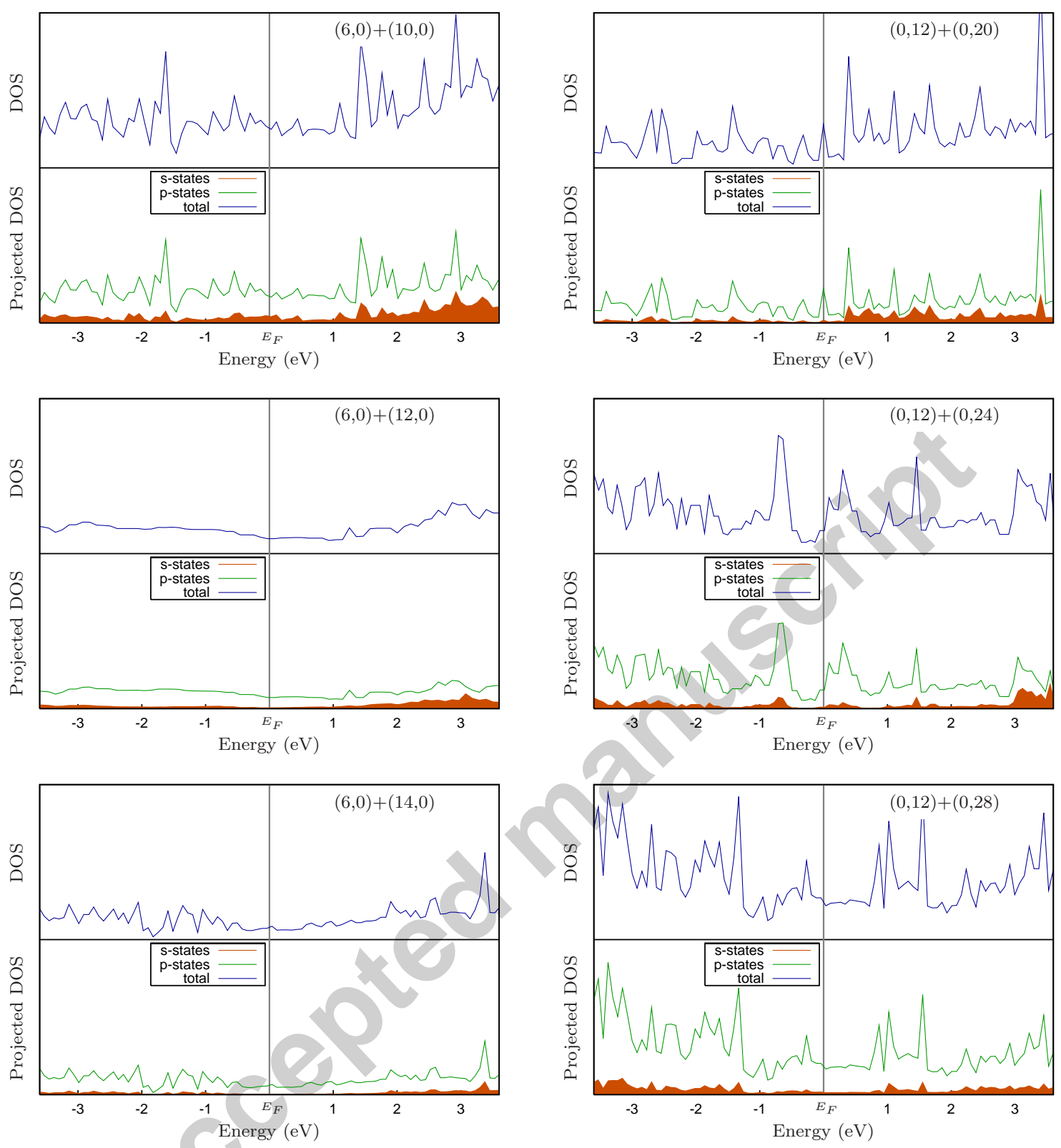

FIG. 6: The total and $l$-decomposed DOS for the DWBNTs. Both total and projected density of states are shown in arbitrary units. 

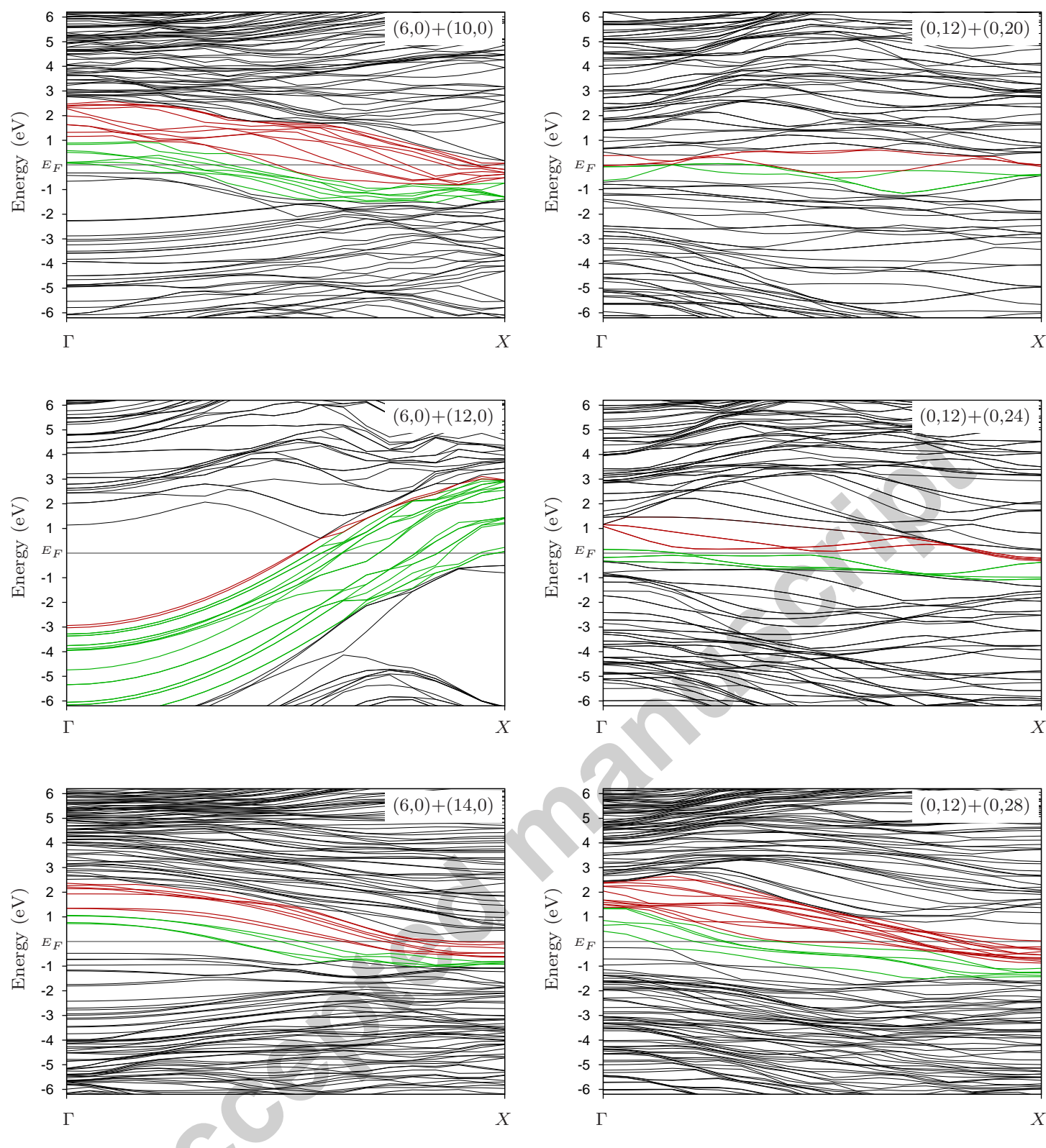

FIG. 7: Calculated band structures of the DWBNTs. Fermi energy level $(0.0 \mathrm{eV})$ is indicated by a straight thick line (gray online). Green and red lines (online) represent the valence and conduction bands, respectively. 